\title{
On the de-Rham Cohomology of Hyperelliptic Curves
}

\author{
Bernhard Köck and Joseph TAit
}

\begin{abstract}
For any hyperelliptic curve $X$, we give an explicit basis of the first de-Rham cohomology of $X$ in terms of Čech cohomology. We use this to produce a family of curves in characteristic $p>2$ for which the Hodgede-Rham short exact sequence does not split equivariantly; this generalises a result of Hortsch. Further, we use our basis to show that the hyperelliptic involution acts on the first de-Rham cohomology by multiplication by -1 , i.e., acts as the identity when $p=2$.
\end{abstract}

MSC-class: 14F40, 14G17, 14H37.

Keywords: hyperelliptic curve; de-Rham cohomology; Čech cohmology; Hodge-de-Rham short exact sequence; hyperelliptic involution.

\section{Introduction}

Recall that the de-Rham cohomology $H_{\mathrm{dR}}^{*}(X / k)$ of a smooth projective curve $X$ over an algebraically closed field $k$ is defined as the hypercohomology of the de-Rham complex

$$
\mathcal{O}_{X} \stackrel{d}{\rightarrow} \Omega_{X}
$$

where $d$ denotes the usual differential map $f \mapsto d f$. In particular, we have a long exact sequence relating $H_{\mathrm{dR}}^{*}(X / k)$ to ordinary cohomology of the structure sheaf $\mathcal{O}_{X}$ and of the sheaf $\Omega_{X}$ of differentials on $X$. The very general and famous fact that the Hodge-de-Rham spectral sequence degenerates at $E_{1}$ (e.g., see [Wed08]) means for our curve $X$ that the following main part of that long sequence is a short exact sequence, see Propositon 2.1.

$$
0 \rightarrow H^{0}\left(X, \Omega_{X}\right) \rightarrow H_{\mathrm{dR}}^{1}(X / k) \rightarrow H^{1}\left(X, \mathcal{O}_{X}\right) \rightarrow 0 .
$$

We call this sequence the Hodge-de-Rham short exact sequence. In particular, the vector space $H_{\mathrm{dR}}^{1}(X / K)$ is the direct sum of the vector spaces $H^{0}\left(X, \Omega_{X}\right)$ and $H^{1}\left(X, \mathcal{O}_{X}\right)$ over $k$.

We now assume furthermore that a finite group $G$ acts on our curve $X$. If $p:=\operatorname{char}(k)$ does not divide the order of $G$, Maschke's Theorem implies 
that the Hodge-de-Rham short exact sequence also splits as a sequence of modules over the group ring $k[G]$.

However, the latter fact fails to be true in general when $p>0$ does divide $\operatorname{ord}(G)$. A counterexample has been constructed in the recent paper [Hor12] by Hortsch. The main goal of this paper is to generalise that counterexample. More precisely, we will prove the following theorem, see Theorem 3.3 .

Theorem. Let $p \geq 3$ and let $q(z) \in k[z]$ be a monic polynomial of odd degree without repeated roots. Let $X$ denote the hyperelliptic curve over $k$ defined by the equation $y^{2}=q\left(x^{p}-x\right)$ and let $G$ denote the subgroup of Aut $(X)$ generated by the automorphism $\tau$ given by $(x, y) \mapsto(x+1, y)$. Then the Hodge-de-Rham short exact sequence does not split as a sequence of $k[G]$ modules.

Beyond [Hor12], this theorem shows (see Remark 3.4) that, for every algebraically closed field $k$ of characteristic $p \geq 3$, there exist infinitely many $g \geq 2$ and hyperelliptic curves $X$ over $k$ of genus $g$ for which the Hodgede-Rham short exact sequence does not split equivariantly. It also shows that, for every $g \geq 2$, there exists a prime $p \geq 3$ and hyperelliptic curves in characteristic $p$ of genus $g$ for which the Hodge-de-Rham short exact sequence does not split equivariantly.

In Example 3.6 and Remark 3.7 we show, using the modular curve $X_{0}(22)$ for $p=3$, that, without assuming the degree of $q(x)$ to be odd, this theorem may be false.

To prove our main theorem, we give an explicit basis of $H_{\mathrm{dR}}^{1}(X / k)$ in terms of Čech cohomology for every hyperelliptic curve $X$, see Theorem 2.2 , and study the action of $\tau$ on that basis.

We provide a basis of $H_{\mathrm{dR}}^{1}(X / k)$ also when $p=2$ and use this to show that the hyperelliptic involution acts trivially on $H_{\mathrm{dR}}^{1}(X / k)$ when $p=2$. In fact, the hyperelliptic involution acts on $H_{\mathrm{dR}}^{1}(X / k)$ by multiplication by -1 for all $p$, see Theorem 3.1 .

If $p=2$, Elkin and Pries construct a subtler basis of $H_{\mathrm{dR}}^{1}(X / k)$ in [EP13] which is suitable to study the action of Frobenius and Verschiebung and, finally, to determine the Ekedahl-Oort type. 


\section{Preliminaries}

In this section, we introduce assumptions and notations used throughout this paper and collect and prove some auxiliary results.

We assume that $k$ is an algebraically closed field of characteristic $p \geq 0$ and that $X$ is a hyperelliptic curve over $k$ of genus $g \geq 2$. We recall that a curve (always assumed to be smooth, projective and irreducible in this paper) is hyperelliptic if there exists a finite, separable morphism of degree two from the curve to $\mathbb{P}_{k}^{1}$. We fix such a map

$$
\pi: X \rightarrow \mathbb{P}_{k}^{1}
$$

which is unique up to automorphisms of $X$ and of $\mathbb{P}_{k}^{1}$ (see [Liu02, Remark 7.4.30]). Let $K(X) / K\left(\mathbb{P}_{k}^{1}\right)=k(x)$ denote the extension of function fields corresponding to $\pi$. According to [Liu02, Proposition 7.4.24 and Remark 7.4.25], we may and will furthermore assume the following concrete description of $K(X)$.

If $p \neq 2$, then $K(X)=k(x, y)$ where $y$ satisfies

$$
y^{2}=f(x)
$$

for some monic polynomial $f(x) \in k[x]$ which has no repeated roots; moreover, $f(x)$ is of degree $2 g+1$ if $\infty \in \mathbb{P}_{k}^{1}$ is a branch point of $\pi$ and of degree $2 g+2$ otherwise. The branch points of $\pi$ are then the roots of $f(x)$, together with $\infty \in \mathbb{P}_{k}^{1}$ if $\operatorname{deg}(f(x))=2 g+1$.

If $p=2$, then $K(X)=k(x, y)$ where $y$ satisfies

$$
y^{2}-h(x) y=f(x)
$$

for some polynomials $h(x), f(x) \in k[x]$ such that $h^{\prime}(x)^{2} f(x)+f^{\prime}(x)^{2}$ and $h(x)$ have no common roots in $k$; moreover, we have $d:=\operatorname{deg}(h(x)) \leq g+1$, with equality if and only if $\infty$ is not a branch point of $\pi$. The branch points of $\pi$ are the roots of $h(x)$, together with $\infty \in \mathbb{P}_{k}^{1}$ if $d<g+1$.

The following estimate for the order of $y$ above $\infty$ is true for both $p \neq 2$ and $p=2$.

Lemma 1.1. Let $P \in \pi^{-1}(\infty)$. Then we have:

$$
\operatorname{ord}_{P}(y) \geq \begin{cases}-(g+1) & \text { if } \pi \text { is unramified at } P \\ -2(g+1) & \text { if } \pi \text { is ramified at } P .\end{cases}
$$


Proof. This is [KT15, Inequality (5.2)].

Lemma 1.2. If $p \neq 2$, let $\omega:=\frac{d x}{y}$ and, if $p=2$, let $\omega:=\frac{d x}{h(x)}$. Then the differentials $\omega, x \omega, \ldots, x^{g-1} \omega$ form a basis of the $k$-vector space $H^{0}\left(X, \Omega_{X}\right)$ of global holomorphic differentials on $X$.

Proof. This is [Liu02, Proposition 7.4.26].

Remark 1.3. A different basis of $H^{0}\left(X, \Omega_{X}\right)$ is given in [Ma78, Lemma 5].

Lemma 1.4. Let $p=2$ and let $P \in \pi^{-1}(\infty)$. Then we have:

$$
\operatorname{ord}_{P}(d x)= \begin{cases}-2 & \text { if } \pi \text { is unramified at } P \\ 2(g-1-d) & \text { if } \pi \text { is ramified at } P .\end{cases}
$$

Proof. By the Riemann-Hurwitz formula [Sti93, Theorem 3.4.6] we have

$$
\operatorname{ord}_{P}(d x)=e_{P} \cdot \operatorname{ord}_{\infty}(d x)+\delta_{P}
$$

where $e_{P}$ denotes the ramification index of $\pi$ at $P$ and $\delta_{P}$ denotes the order of the ramification divisor of $\pi$ at $P$. It is easy to see that $\operatorname{ord}_{\infty}(d x)=-2$. Therefore $\operatorname{ord}_{P}(d x)=-2$ if $\pi$ is unramified at $P$. On the other hand, if $\pi$ is ramified at $P$, we have $\delta_{P}=2(g+1-d)$ by [KT15, Equation (5.3)] and hence

$$
\operatorname{ord}_{P}(d x)=2 \cdot(-2)+2(g+1-d)=2(g-1-d),
$$

as claimed.

We define $U_{a}=X \backslash \pi^{-1}(a)$ for any $a \in \mathbb{P}_{k}^{1}$ and let $\mathcal{U}$ be the affine cover of $X$ formed by $U_{0}$ and $U_{\infty}$. Given any sheaf $\mathcal{F}$ on $X$ we have the Cech differential $\check{d}: \mathcal{F}\left(U_{0}\right) \times \mathcal{F}\left(U_{\infty}\right) \rightarrow \mathcal{F}\left(U_{0} \cap U_{\infty}\right)$, defined by $\left.\left(f_{0}, f_{\infty}\right) \mapsto f_{0}\right|_{U_{0} \cap U_{\infty}}-\left.f_{\infty}\right|_{U_{0} \cap U_{\infty}}$. In general we will suppress the notation denoting the restriction map. The first cohomology group $\frac{\mathcal{O}_{X}\left(U_{0} \cap U_{\infty}\right)}{\operatorname{Im}(d)}$ of the cochain complex

$$
0 \rightarrow \mathcal{O}_{X}\left(U_{0}\right) \times \mathcal{O}_{X}\left(U_{\infty}\right) \stackrel{\check{d}}{\rightarrow} \mathcal{O}_{X}\left(U_{0} \cap U_{\infty}\right) \rightarrow 0 .
$$

is the first Cech cohomology group $\check{H}^{1}\left(\mathcal{U}, \mathcal{O}_{X}\right)$. By Leray's theorem Liu02, Theorem 5.2.12] and Serre's affineness criterion [Liu02, Theorem 5.2.23] we therefore have

$$
H^{1}\left(X, \mathcal{O}_{X}\right) \cong \frac{\mathcal{O}_{X}\left(U_{0} \cap U_{\infty}\right)}{\left\{f_{0}-f_{\infty} \mid f_{0} \in \mathcal{O}_{X}\left(U_{0}\right), f_{\infty} \in \mathcal{O}_{X}\left(U_{\infty}\right)\right\}} .
$$

When describing elements of $H^{1}\left(X, \mathcal{O}_{X}\right)$ using this isomorphisms we will denote the residue class of $f \in \mathcal{O}_{X}\left(U_{0} \cap U_{\infty}\right)$ by $[f]$. 
Proposition 1.5. The elements $\frac{y}{x}, \ldots, \frac{y}{x^{g}} \in K(X)$ are regular on $U_{0} \cap U_{\infty}$, and their residue classes $\left[\frac{y}{x}\right], \ldots,\left[\frac{y}{x^{g}}\right]$ form a basis of $H^{1}\left(X, \mathcal{O}_{X}\right)$.

Proof. By [Liu02, Proposition 7.4.24(b)], we may identify $\mathcal{O}_{X}\left(U_{\infty}\right)$ with the $k$-algebra $k[x, y]$ defined by the relation given in (1) or (2). Then $\mathcal{O}_{X}\left(U_{0} \cap U_{\infty}\right)$ is $k\left[x^{ \pm 1}, y\right]$. As the relations in (1) and (2) are quadratic in $y$, the elements $\ldots, \frac{1}{x^{2}}, \frac{1}{x}, 1, x, x^{2}, \ldots$ and $\ldots, \frac{y}{x^{2}}, \frac{y}{x}, y, x y, x^{2} y, \ldots$ form a $k$-basis of $k\left[x^{ \pm 1}, y\right]$. The elements $1, x, x^{2}, \ldots$ and $y, x y, x^{2} y, \ldots$ obviously form a basis of the image of $\mathcal{O}_{X}\left(U_{\infty}\right)$ in $\mathcal{O}_{X}\left(U_{0} \cap U_{\infty}\right)$. By [Liu02, Proposition 7.4.24(b)], the image of $\mathcal{O}_{X}\left(U_{0}\right)$ in $\mathcal{O}_{X}\left(U_{0} \cap U_{\infty}\right)$ consists of elements of the form $g\left(\frac{1}{x}, \frac{y}{x^{g+1}}\right)$ where $g \in k[s, t]$. Hence, the elements $\ldots, \frac{1}{x^{2}}, \frac{1}{x}, 1$ and $\ldots \frac{y}{x^{g+3}}, \frac{y}{x^{g+2}}, \frac{y}{x^{g+1}}$ form a basis of that image. We conclude that the residue classes $\left[\frac{y}{x}\right], \ldots,\left[\frac{y}{x^{g}}\right]$ form a basis of $H^{1}\left(X, \mathcal{O}_{X}\right)$, as was to be shown.

Remark 1.6. Let $\omega_{j}:=\frac{x^{j-1}}{y} d x$ when $p \neq 2$ and let $\omega_{j}=\frac{x^{j-1}}{h(x)} d x$ when $p=2$. Then, by Lemma 1.2 , the elements $\omega_{j}, j=1, \ldots, g$, form a $k$-basis of $H^{0}\left(X, \Omega_{X}\right)$. Let $\langle\rangle:, H^{0}\left(X, \Omega_{X}\right) \times H^{1}\left(X, \mathcal{O}_{X}\right) \rightarrow k$ denote Serre duality. Then $\left\langle\omega_{j},\left[\frac{y}{x^{i}}\right]\right\rangle$ vanishes if $j \neq i$ and is non-zero if $j=i$, see the proof of [Tai14, Theorem 4.2.1]. In other words, up to multiplication by scalars, the basis $\left[\frac{y}{x^{i}}\right], i=1, \ldots, g$, of $H^{1}\left(X, \mathcal{O}_{X}\right)$, given in Proposition 1.5 . is dual to the basis $\omega_{j}, j=1, \ldots, g$, with respect to Serre duality.

Remark 1.7. Different bases of $H^{1}\left(X, \mathcal{O}_{X}\right)$ are described in [Su75, Lemma 6] and [Ma78, Lemma 6].

\section{Bases of $H_{\mathrm{dR}}^{1}(X / k)$}

The object of this section is to give an explicit $k$-basis for the first de-Rham cohomology group $H_{\mathrm{dR}}^{1}(X / k)$ using Čech cohomology. If $p \neq 2$, we will moreover refine our result when another open subset is added to our standard open cover of $X$.

The algebraic de-Rham cohomology of $X$ is defined to be the hypercohomology of the de-Rham complex

$$
0 \rightarrow \mathcal{O}_{X} \stackrel{d}{\rightarrow} \Omega_{X} \rightarrow 0
$$

where $d$ denotes the usual differential map $f \mapsto d f$. We use the cover $\mathcal{U}$ and the Čech differentials defined in the previous section to obtain the Čech 
bicomplex of (5):

(6)
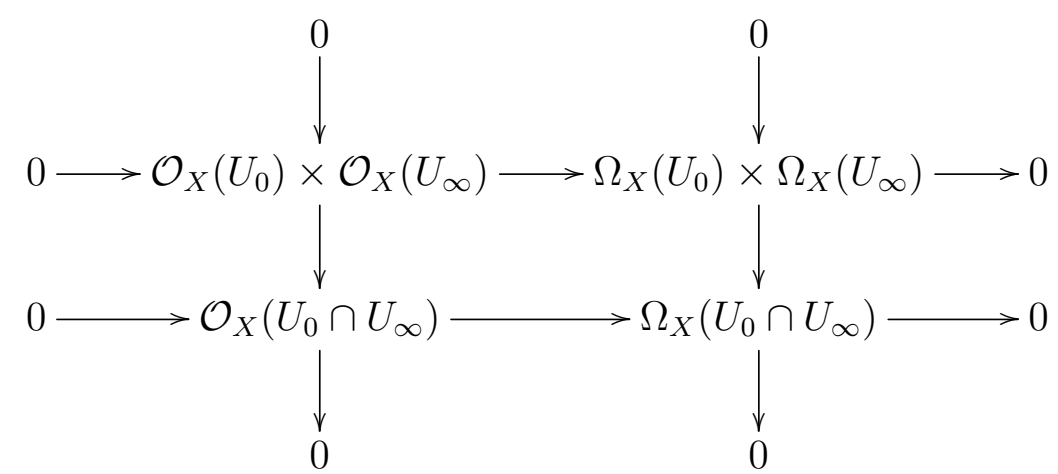

By a generalisation of Leray's theorem [Gro61, Corollaire 12.4.7] and Serre's affineness criterion [Liu02, Theorem 5.2.23], the first de-Rham cohomology of $X$ is isomorphic to the first cohomology of the total complex of (6). Thus, $H_{\mathrm{dR}}^{1}(X / k)$ is isomorphic to the quotient of the space

$$
\begin{aligned}
\left\{\left(\omega_{0}, \omega_{\infty}, f_{0 \infty}\right) \in \Omega_{X}\left(U_{0}\right) \times \Omega_{X}\left(U_{\infty}\right) \times \mathcal{O}_{X}\left(U_{0} \cap U_{\infty}\right) \mid\right. \\
\left.d f_{0 \infty}=\left.\omega_{0}\right|_{U_{0} \cap U_{\infty}}-\left.\omega_{\infty}\right|_{U_{0} \cap U_{\infty}}\right\}
\end{aligned}
$$

by the subspace

$$
\left\{\left(d f_{0}, d f_{\infty},\left.f_{0}\right|_{U_{0} \cap U_{\infty}}-\left.f_{\infty}\right|_{U_{0} \cap U_{\infty}}\right) \mid f_{0} \in \mathcal{O}_{X}\left(U_{0}\right), f_{\infty} \in \mathcal{O}_{X}\left(U_{\infty}\right)\right\} .
$$

Via this representation of $H_{\mathrm{dR}}^{1}(X / k)$ and the isomorphism (4) we obtain the canonical maps

$$
i: H^{0}\left(X, \Omega_{X}\right) \rightarrow H_{\mathrm{dR}}^{1}(X / k), \quad \omega \mapsto\left[\left(\left.\omega\right|_{U_{0}},\left.\omega\right|_{U_{\infty}}, 0\right)\right]
$$

and

$$
p: H_{\mathrm{dR}}^{1}(X / k) \rightarrow H^{1}\left(X, \mathcal{O}_{X}\right), \quad\left[\left(\omega_{0}, \omega_{\infty}, f_{0 \infty}\right)\right] \mapsto\left[f_{0 \infty}\right] .
$$

+ The following proposition is equivalent to the more familiar and fancier sounding statement that the Hodge-de-Rham spectral sequence for $X$ degenerates at $E_{1}$ (see [Wed08]) and is in fact true for every smooth, proper curve $X$ over $k$, see example (2) in section (1.5) of [Wed08].

Proposition 2.1. The following sequence is exact:

$$
0 \rightarrow H^{0}\left(X, \Omega_{X}\right) \stackrel{i}{\rightarrow} H_{\mathrm{dR}}^{1}(X / k) \stackrel{p}{\rightarrow} H^{1}\left(X, \mathcal{O}_{X}\right) \rightarrow 0 .
$$


We will call the sequence (11) the Hodge-de-Rham short exact sequence.

An elementary proof of Proposition 2.1 (that works for every smooth projective curve) can be found in [Tai14, Proposition 4.1.2]; the main ingredient there is just the fact that the residue of differentials of the form $d f$ vanishes at every point of $X$ and that hence the obvious composition $H^{1}\left(X, \mathcal{O}_{X}\right) \rightarrow$ $H^{1}\left(X, \Omega_{X}\right) \stackrel{\sim}{\rightarrow} k$ is the zero map. For a hyperelliptic curve $X$, the surjectivity of $p$ will also be verified in the proof of Theorem 2.2 below.

In order to state a basis of $H_{\mathrm{dR}}^{1}(X / k)$, we now define certain polynomials. To this end, we introduce the notations $f^{\leq m}(x):=a_{0}+\ldots+a_{m} x^{m}$ and $f^{>m}(x):=a_{m+1} x^{m+1}+\ldots+a_{n} x^{n}$ for any polynomial $f(x):=a_{0}+\ldots+a_{n} x^{n} \in$ $k[x]$ and any $m \geq 0$. Let $1 \leq i \leq g$.

When $p \neq 2$ we define

$$
s_{i}(x):=x f^{\prime}(x)-2 i f(x) \in k[x]
$$

and put $\psi_{i}(x):=s_{i}^{\leq i}(x)$ and $\phi_{i}(x):=s_{i}^{>i}(x)$ so that $s_{i}(x)=\psi_{i}(x)+\phi_{i}(x)$.

When $p=2$ we define

$$
s_{i}(x, y):=x f^{\prime}(x)+\left(x h^{\prime}(x)+i h(x)\right) y \in k[x] \oplus k[x] y \subseteq k(x, y)
$$

(where $k[x] \oplus k[x] y$ denotes the $k[x]$-module generated by 1 and $y$ ) and put $\psi_{i}(x, y):=s_{i}^{\leq i}(x, y)$ and $\phi_{i}(x, y):=s_{i}^{>i}(x, y)$ where now the operations $\leq i$ and $>i$ are applied to both the coefficients $x f^{\prime}(x)$ and $x h^{\prime}(x)+i h(x)$. Again we have $s_{i}(x, y)=\psi_{i}(x, y)+\phi_{i}(x, y)$.

We now give a basis of $H_{\mathrm{dR}}^{1}(X / k)$ in terms of the polynomials just introduced and using the presentation of $H_{\mathrm{dR}}^{1}(X / k)$ developed above.

Theorem 2.2. If $p \neq 2$, the residue classes

$$
\gamma_{i}:=\left[\left(\frac{\psi_{i}(x)}{2 x^{i+1} y} d x, \frac{-\phi_{i}(x)}{2 x^{i+1} y} d x, \frac{y}{x^{i}}\right)\right], \quad i=1, \ldots, g
$$

along with the residue classes

$$
\lambda_{i}:=\left[\left(\frac{x^{i}}{y} d x, \frac{x^{i}}{y} d x, 0\right)\right], \quad i=0, \ldots, g-1,
$$

form a $k$-basis of $H_{\mathrm{dR}}^{1}(X / k)$. 
On the other hand, if $p=2$, the residue classes

$$
\gamma_{i}:=\left[\left(\frac{\psi_{i}(x, y)}{x^{i+1} h(x)} d x, \frac{\phi_{i}(x, y)}{x^{i+1} h(x)} d x, \frac{y}{x^{i}}\right)\right], \quad i=1, \ldots, g,
$$

together with the residue classes

$$
\lambda_{i}:=\left[\left(\frac{x^{i}}{h(x)} d x, \frac{x^{i}}{h(x)} d x, 0\right)\right], \quad i=0, \ldots, g-1,
$$

form a $k$-basis of $H_{\mathrm{dR}}^{1}(X / k)$.

Proof. The elements in (13) and (15) are the images under the map $i$ of the differentials $\frac{x^{i}}{y} d x, i=0, \ldots, g-1$, and $\frac{x^{i}}{h(x)} d x, i=0, \ldots, g-1$, respectively, which form a basis of $H^{0}\left(X, \Omega_{X}\right)$ by Lemma 1.2. Furthermore, provided the elements in $(12)$ and $(14)$ are well-defined elements of $H_{\mathrm{dR}}^{1}(X / k)$, these elements are mapped to the elements $\left[\frac{y}{r^{i}}\right], i=1, \ldots, g$, under $p$, which form a basis of $H^{1}\left(X, \mathcal{O}_{X}\right)$ by Proposition 1.5. By Proposition 2.1, it therefore suffices to check that the elements in (12) and (14) are well-defined elements of $H_{\mathrm{dR}}^{1}(X / k)$.

We first check the equality in (7). When $p \neq 2$, this is verified as follows:

$$
\begin{gathered}
\left(\frac{\psi_{i}(x)}{2 x^{i+1} y}-\frac{-\phi_{i}(x)}{2 x^{i+1} y}\right) d x=\frac{s_{i}(x)}{2 x^{i+1} y} d x \\
=\frac{x f^{\prime}(x)-2 i f(x)}{2 x^{i+1} y} d x=\frac{x^{i}}{2 y}\left(\frac{f^{\prime}(x)}{x^{2 i}}-\frac{2 i f(x)}{x^{2 i+1}}\right) d x \\
=\frac{x^{i}}{2 y} d\left(\frac{f(x)}{x^{2 i}}\right)=\frac{x^{i}}{2 y} d\left(\left(\frac{y}{x^{i}}\right)^{2}\right)=d\left(\frac{y}{x^{i}}\right) .
\end{gathered}
$$

When $p=2$, we obtain

$$
h^{\prime}(x) y d x+h(x) d y=f^{\prime}(x) d x
$$

by differentiating equation (2) and then verify the equality in (7) as follows (note that we replace all minus signs with plus signs):

$$
\begin{gathered}
\left(\frac{\psi_{i}(x, y)}{x^{i+1} h(x)}+\frac{\phi_{i}(x, y)}{x^{i+1} h(x)}\right) d x=\frac{s_{i}(x, y)}{x^{i+1} h(x)} d x \\
=\left(\frac{f^{\prime}(x)}{x^{i} h(x)}+\frac{h^{\prime}(x) y}{x^{i} h(x)}+\frac{i y}{x^{i+1}}\right) d x \\
=\frac{d y}{x^{i}}+\frac{i y}{x^{i+1}} d x=d\left(\frac{y}{x^{i}}\right) .
\end{gathered}
$$


It remains to prove that the first two entries of the triples in $(12)$ and $(14)$ are regular differentials on $U_{0}$ and $U_{\infty}$, respectively.

We first consider the case $p \neq 2$. As $\frac{d x}{y}$ is a regular differential on $X=$ $U_{0} \cup U_{\infty}$ by Lemma 1.2 , it suffices to observe that each of the functions $\frac{\psi_{i}(x)}{x^{i+1}}$, $i=1, \ldots, g$, is regular on $U_{0}$ (in fact has a zero at $\infty$ ) and that each of the functions $\frac{\phi_{i}(x)}{x^{i+1}}, i=1, \ldots, g$, is regular on $U_{\infty}$.

We now turn to the case $p=2$. As above, we know from Lemma 1.2 that $\frac{d x}{h(x)}$ is regular on $X=U_{0} \cup U_{\infty}$. Furthermore, for every $i \in\{1, \ldots, g\}$, the function $\frac{\phi_{i}(x, y)}{x^{i+1}}$ is regular on $U_{\infty}$ since $y$ is regular on $U_{\infty}$ and since, by definition of $\phi_{i}(x, y)$, the $k[x]$-coefficients of 1 and $y$ in $\phi_{i}(x, y)$ are divisible by $x^{i+1}$. Hence $\frac{\phi_{i}(x, y)}{x^{i+1} h(x)} d x$ is regular on $U_{\infty}$, as was to be shown. It remains to show that $\frac{\psi_{i}(x, y)}{x^{i+1} h(x)} d x$ is regular on $U_{0}$. As $\frac{\psi_{i}(x, y)}{x^{i+1}}$ and $\frac{d x}{h(x)}$ are regular on $U_{0} \cap U_{\infty}$, this amounts to showing that $\frac{\psi_{i}(x, y)}{x^{i+1} h(x)} d x$ is regular above $\infty$.

We first consider the case when $\infty$ is not a branch point of $\pi$. By Lemma 1.4, the differential $d x$ has a pole of order 2 at each of the two points $P_{\infty}, P_{\infty}^{\prime} \in X$ above $\infty$. Furthermore, the $k[x]$-coefficient of 1 in $\psi_{i}(x, y)$ has a pole at $P_{\infty}$ and $P_{\infty}^{\prime}$ of order at most $i$ and the $k[x]$-coefficient of $y$ has a pole at $P_{\infty}$ and $P_{\infty}^{\prime}$ of order at most $i-1$ since the coefficient of $x^{i}$ in $x h^{\prime}(x)+i h(x)$ is zero. Moreover, $y$ has a pole at $P_{\infty}$ and $P_{\infty}^{\prime}$ of order at most $g+1$ by Lemma 1.1. Finally, $\frac{1}{h(x)}$ has a zero at $P_{\infty}$ and $P_{\infty}^{\prime}$ of order $d=\operatorname{deg}(h(x))=g+1$. Putting all this together we obtain

$$
\begin{aligned}
& \operatorname{ord}_{P}\left(\frac{\psi_{i}(x, y)}{x^{i+1} h(x)} d x\right) \\
& \quad=\operatorname{ord}_{P}\left(\psi_{i}(x, y)\right)+\operatorname{ord}_{P}\left(\frac{1}{x^{i+1}}\right)+\operatorname{ord}_{P}\left(\frac{1}{h(x)}\right)+\operatorname{ord}_{P}(d x) \\
& \quad \geq \min \{-i,-(i-1)-(g+1)\}+(i+1)+(g+1)-2=0
\end{aligned}
$$

for $P \in\left\{P_{\infty}, P_{\infty}^{\prime}\right\}$, which shows that $\frac{\psi_{i}(x, y)}{x^{i+1} h(x)} d x$ is regular at $P_{\infty}$ and $P_{\infty}^{\prime}$.

We finally assume that $\infty$ is a branch point of $\pi$ and prove that $\frac{\psi_{i}(x, y)}{x^{i+1} h(x)} d x$ is regular at the unique point $P_{\infty} \in X$ above $\infty$. By Lemma 1.4, the order of the differential $d x$ at $P_{\infty}$ is $2(g-1-d)$ where $d=\operatorname{deg}(h(x))$. For similar reasons as above, the $k[x]$-coefficients of 1 and $y$ in $\psi_{i}(x, y)$ have a pole at $P_{\infty}$ of order at most $2 i$ and $2(i-1)$, respectively, and $\frac{1}{h(x)}$ has a zero at $P_{\infty}$ of order $2 d$. Finally, $y$ has a pole at $P_{\infty}$ of order at most $2(g+1)$ by Lemma 1.1 . 
Putting all this together we obtain

$$
\begin{aligned}
& \operatorname{ord}_{P_{\infty}}\left(\frac{\psi_{i}(x, y)}{x^{i+1} h(x)} d x\right) \\
& \quad=\operatorname{ord}_{P_{\infty}}\left(\psi_{i}(x, y)\right)+\operatorname{ord}_{P_{\infty}}\left(\frac{1}{x^{i+1}}\right)+\operatorname{ord}_{P_{\infty}}\left(\frac{1}{h(x)}\right)+\operatorname{ord}_{P_{\infty}}(d x) \\
& \quad \geq \min \{-2 i,-2(i-1)-2(g+1)\}+2(i+1)+2 d+2(g-1-d)=0,
\end{aligned}
$$

which shows that $\frac{\psi_{i}(x, y)}{x^{i+1} h(x)} d x$ is regular at $P_{\infty}$.

In the proofs in the next section, we will need a refined description of the basis elements given in (12) when another open subset is added to our standard cover $\mathcal{U}=\left\{U_{0}, U_{\infty}\right\}$. To this end, we now fix $a \in \mathbb{P}_{k}^{1} \backslash\{0, \infty\}$ and define the covers $\mathcal{U}^{\prime}:=\left\{U_{a}, U_{\infty}\right\}$ and $\mathcal{U}^{\prime \prime}:=\left\{U_{0}, U_{a}, U_{\infty}\right\}$ of $X$. Similarly to (7) and (8), the first de-Rham cohmology group $H_{\mathrm{dR}}^{1}(X / k)$ is then isomorphic to the $k$-vector space

(16) $\left\{\left(\omega_{0}, \omega_{a}, \omega_{\infty}, f_{0 a}, f_{0 \infty}, f_{a \infty}\right) \in\right.$ $\Omega_{X}\left(U_{0}\right) \times \Omega_{X}\left(U_{a}\right) \times \Omega_{X}\left(U_{\infty}\right) \times \mathcal{O}_{X}\left(U_{0} \cap U_{a}\right) \times \mathcal{O}_{X}\left(U_{0} \cap U_{\infty}\right) \times \mathcal{O}_{X}\left(U_{a} \cap U_{\infty}\right) \mid$ $\left.f_{0 a}-f_{0 \infty}+f_{a \infty}=0, d f_{0 a}=\omega_{0}-\omega_{a}, d f_{0 \infty}=\omega_{0}-\omega_{\infty}, d f_{a \infty}=\omega_{a}-\omega_{\infty}\right\}$

quotiented by the subspace

$$
\begin{aligned}
& \left\{\left(d f_{0}, d f_{a}, d f_{\infty}, f_{0}-f_{a}, f_{0}-f_{\infty}, f_{a}-f_{\infty}\right) \mid\right. \\
& \left.\quad f_{0} \in \mathcal{O}_{X}\left(U_{0}\right), f_{a} \in \mathcal{O}_{X}\left(U_{a}\right), f_{\infty} \in \mathcal{O}_{X}\left(U_{\infty}\right)\right\}
\end{aligned}
$$

We use the notations $\check{H}_{\mathrm{dR}}^{1}(\mathcal{U})$ and $\check{H}_{\mathrm{dR}}^{1}\left(\mathcal{U}^{\prime \prime}\right)$ for the representations of $H_{\mathrm{dR}}^{1}(X / k)$ introduced in (7), (8) and $(16),(17)$, respectively. The canonical isomorphism $\rho: \check{H}_{\mathrm{dR}}^{1}\left(\mathcal{U}^{\prime \prime}\right) \rightarrow \check{H}_{\mathrm{dR}}^{1}(\mathcal{U})$, is then induced by the projection

$$
\rho:\left(\omega_{0}, \omega_{a}, \omega_{\infty}, f_{0 a}, f_{0 \infty}, f_{a \infty}\right) \mapsto\left(\omega_{0}, \omega_{\infty}, f_{0 \infty}\right) .
$$

When $p \neq 2$, the next proposition explicitly describes the pre-image of the basis elements $\gamma_{i}=\left[\left(\frac{\psi_{i}(x)}{2 x^{i+1} y} d x, \frac{-\phi_{i}(x)}{2 x^{i+1} y} d x, \frac{y}{x^{i}}\right)\right], i=1, \ldots, g$, of $H_{\mathrm{dR}}^{1}(X / k)$ under $\rho$. To this end, we define the polynomials

$$
g(x):=(x-a)^{g}, \quad r_{i}(x):=g^{\leq i-1}(x) \quad \text { and } \quad t_{i}(x):=g^{>i-1}(x)
$$

in $k[x]$ for $1 \leq i \leq g$ so that $r_{i}(x)+t_{i}(x)=(x-a)^{g}$. 
Proposition 2.3. Let $p \neq 2$. For $i \in\{1, \ldots, g\}$, let

$$
\begin{gathered}
\omega_{0 i}:=\frac{\psi_{i}(x)}{2 x^{i+1} y} d x, \quad \omega_{\infty i}:=\frac{-\phi_{i}(x)}{2 x^{i+1} y} d x \\
\omega_{a i}:=\frac{\left(\psi_{i}(x) t_{i}(x)-\phi_{i}(x) r_{i}(x)\right)(x-a)-2 i f(x)(-1)^{g-i}\left(\begin{array}{c}
g \\
i
\end{array}\right) a^{g-i+1} x^{i}}{2 x^{i+1}(x-a)^{g+1} y} d x
\end{gathered}
$$

and

$$
f_{0 a i}:=\frac{r_{i}(x) y}{x^{i}(x-a)^{g}}, \quad f_{0 \infty i}:=\frac{y}{x^{i}}, \quad f_{a \infty i}:=\frac{t_{i}(x) y}{x^{i}(x-a)^{g}} .
$$

Then we have:

$$
\rho^{-1}\left(\gamma_{i}\right)=\left[\left(\omega_{0 i}, \omega_{a i}, \omega_{\infty i}, f_{0 a i}, f_{0 \infty i}, f_{a \infty i}\right)\right]
$$

Proof. We fix $i \in\{1, \ldots, g\}$. We obviously only need to show that the sextuple on the right-hand side of $(19)$ is a well-defined element of the space (16).

From the proof of Theorem 2.2 we already know that $d\left(f_{0 \infty i}\right)=\omega_{0 i}-\omega_{\infty i}$ and that $f_{0 \infty i}, \omega_{0 i}$ and $\omega_{\infty i}$ are regular on the appropriate open sets.

Since $r_{i}(x)+t_{i}(x)=(x-a)^{g}$, we have

$$
f_{0 a i}-f_{0 \infty i}+f_{a \infty i}=\frac{r_{i}(x) y}{x^{i}(x-a)^{g}}-\frac{y}{x^{i}}+\frac{t_{i}(x) y}{x^{i}(x-a)^{g}}=0,
$$

as desired.

The function $f_{0 a i}$ is obviously regular above $\mathbb{P}_{k}^{1} \backslash\{0, a, \infty\}$. We furthermore observe that $\operatorname{ord}_{\infty}\left(\frac{r_{i}(x)}{x^{i}(x-a)^{g}}\right) \geq-(i-1)+i+g=g+1$ and that, by Lemma 1.1, the order of $y$ above $\infty$ is at least $-2(g+1)$ or at least $-(g+1)$ depending on whether $\infty$ is a branch point of $\pi$ or not. Thus, $f_{0 a i}$ is regular above $\infty$ and hence on $U_{0} \cap U_{a}$.

As above, the function $f_{a \infty i}$ is regular above $\mathbb{P}_{k}^{1} \backslash\{0, a, \infty\}$. Furthermore, the functions $\frac{t_{i}(x)}{x^{i}}, y$ and $\frac{1}{(x-a)^{g}}$ are obviously regular above 0 . Therefore, $f_{a \infty i}$ is regular above 0 as well and hence on $U_{a} \cap U_{\infty}$.

We next show that $d f_{0 a i}=\omega_{0 i}-\omega_{a i}$. Using the product rule and the chain 
rule we obtain

$$
\begin{aligned}
& d f_{0 a i}=d\left(\frac{r_{i}(x) y}{x^{i}(x-a)^{g}}\right) \\
& =\frac{r_{i}(x)}{x^{i}(x-a)^{g}} d y+d\left(\frac{r_{i}(x)}{x^{i}(x-a)^{g}}\right) y \\
& =\frac{f^{\prime}(x) r_{i}(x)}{2 x^{i}(x-a)^{g} y} d x+\left(\frac{r_{i}^{\prime}(x)}{x^{i}(x-a)^{g}}-\frac{i r_{i}(x)}{x^{i+1}(x-a)^{g}}-\frac{g r_{i}(x)}{x^{i}(x-a)^{g+1}}\right) y d x \\
& =\frac{x f^{\prime}(x) r_{i}(x)(x-a)+2 f(x)\left(x r_{i}^{\prime}(x)(x-a)-i r_{i}(x)(x-a)-g x r_{i}(x)\right)}{2 x^{i+1}(x-a)^{g+1} y} d x .
\end{aligned}
$$

We now recall that

$$
x f^{\prime}(x)-2 i f(x)=\psi_{i}(x)+\phi_{i}(x) .
$$

We furthermore recall that $r_{i}(x)=g^{\leq i-1}(x)$ where $g(x)=(x-a)^{g}$. Therefore

$$
\begin{aligned}
r_{i}^{\prime}(x) & \cdot(x-a)-g \cdot r_{i}(x) \\
& =\left[g^{\prime}(x)\right]^{\leq i-2} \cdot(x-a)-g \cdot g^{\leq i-1}(x) \\
& =\left(\left[g^{\prime}(x) \cdot(x-a)\right]^{\leq i-1}+a \cdot b_{i-1} \cdot x^{i-1}\right)-g \cdot g^{\leq i-1}(x) \\
& =a \cdot b_{i-1} \cdot x^{i-1}
\end{aligned}
$$

where $b_{i-1}=(-1)^{g-i} i\left(\begin{array}{c}g \\ i\end{array}\right) a^{g-i}$ denotes the coefficient of $x^{i-1}$ in $g^{\prime}(x)$.

Thus we obtain

$$
\begin{aligned}
& d f_{0 a i}=\frac{\left(\psi_{i}(x)+\phi_{i}(x)\right) r_{i}(x)(x-a)+2 f(x)\left(a b_{i-1} x^{i}\right)}{2 x^{i+1}(x-a)^{g+1} y} d x \\
& =\frac{\psi_{i}(x)\left((x-a)^{g+1}-t_{i}(x)(x-a)\right)+\phi_{i}(x) r_{i}(x)(x-a)+2 f(x)\left(a b_{i-1}\right) x^{i}}{2 x^{i+1}(x-a)^{g+1} y} d x \\
& =\frac{\psi_{i}(x)}{2 x^{i+1} y} d x-\frac{\left(\psi_{i}(x) t_{i}(x)-\phi_{i}(x) r_{i}(x)\right)(x-a)-2 f(x)\left(a b_{i-1}\right) x^{i}}{2 x^{i+1}(x-a)^{g+1} y} d x \\
& =\omega_{0 i}-\omega_{a i},
\end{aligned}
$$

as claimed.

From the above we moreover obtain that

$$
d f_{a \infty i}=d f_{0 \infty i}-d f_{0 a i}=\left(\omega_{0 i}-\omega_{\infty i}\right)-\left(\omega_{0 i}-\omega_{a i}\right)=\omega_{a i}-\omega_{\infty i} .
$$

Finally, $\omega_{a i}$ is regular on $U_{a}$ because $\omega_{a i}=\omega_{0 i}-d f_{0 a i}$ and $\omega_{a i}$ is hence regular on $U_{0} \cap U_{a}$ and because $\omega_{a i}=\omega_{\infty i}+d f_{a \infty i}$ and $\omega_{a i}$ is hence regular on $U_{a} \cap U_{\infty}$. 
Remark 2.4. If $p=2$, another basis of $H_{\mathrm{dR}}^{1}(X / k)$ is given in EP13, Section 4].

\section{Actions on $H_{\mathrm{dR}}^{1}(X / k)$}

In this section we study the action of certain automorphisms on $H_{\mathrm{dR}}^{1}(X / k)$. We first prove that the hyperelliptic involution acts by multiplication by -1 on $H_{\mathrm{dR}}^{1}(X / k)$ when $p \neq 2$ and as the identity when $p=2$. We then give a family of hyperelliptic curves for which the Hodge-de-Rham short exact sequence (11) does not split equivariantly.

Theorem 3.1. The hyperelliptic involution acts on $H_{\mathrm{dR}}^{1}(X / k)$ by multiplication by -1 .

Proof. Recall that the hyperelliptic involution is the unique non-trivial automorphism $\sigma$ of $X$ such that $\pi \circ \sigma=\pi$.

If $p \neq 2$, the involution $\sigma$ acts on $K(X)$ by $(x, y) \mapsto(x,-y)$. Hence, $\sigma$ maps each entry of the triples in 12 and 13 to its negative. Thus, the $p \neq 2$ part of Theorem 2.2 implies Theorem 3.1 .

If $p=2$, the involution $\sigma$ acts on $K(X)$ by $(x, y) \mapsto(x, y+h(x))$. In particular, it fixes the basis elements 15$)$ of $H_{\mathrm{dR}}^{1}(X / k)$. According to the $p=2$ part of Theorem 2.2, it remains to show that $\sigma$ also fixes the residue classes $\left[\left(\omega_{0 i}, \omega_{\infty i}, f_{0 \infty i}\right)\right], i=1, \ldots, g$, in $(14)$. For $i \in\{1, \ldots, g\}$, this follows from the description of $H_{\mathrm{dR}}^{1}(X / k)$ given in (7) and (8) and from the equation

$$
\begin{aligned}
\sigma\left(\left(\omega_{0 i}, \omega_{\infty i}, f_{0 \infty i}\right)\right) & -\left(\omega_{0 i}, \omega_{\infty i}, f_{0 \infty i}\right) \\
& =\left(d\left(\frac{h^{\leq i}(x)}{x^{i}}\right), d\left(\frac{h^{>i}(x)}{x^{i}}\right), \frac{h^{\leq i}(x)}{x^{i}}-\frac{h^{>i}(x)}{x^{i}}\right)
\end{aligned}
$$

which in turn is verified in the following three lines (where we replace all minus signs with plus signs):

$$
\begin{gathered}
\sigma\left(\frac{\psi_{i}(x, y)}{x^{i+1} h(x)} d x\right)+\frac{\psi_{i}(x, y)}{x^{i+1} h(x)} d x=\frac{\left[x h^{\prime}(x)+i h(x)\right]^{\leq i} h(x)}{x^{i+1} h(x)} d x=d\left(\frac{h^{\leq i}(x)}{x^{i}}\right) \\
\sigma\left(\frac{\phi_{i}(x, y)}{x^{i+1} h(x)} d x\right)+\frac{\phi_{i}(x, y)}{x^{i+1} h(x)} d x=\frac{\left[x h^{\prime}(x)+i h(x)\right]^{>i} h(x)}{x^{i+1} h(x)} d x=d\left(\frac{h^{>i}(x)}{x^{i}}\right) \\
\sigma\left(\frac{y}{x^{i}}\right)+\frac{y}{x^{i}}=\frac{h(x)}{x^{i}} .
\end{gathered}
$$


Remark 3.2. If $p \neq 2$, Theorem 3.1 can also be proved as follows. By Lemma 1.2, the involution $\sigma$ acts by multiplication by -1 on $H^{0}\left(X, \Omega_{X}\right)$. By Serre duality, it then acts by multiplication by -1 also on $H^{1}\left(X, \mathcal{O}_{X}\right)$. Finally, by Maschke's Theorem applied to the Hodge-de-Rham short exact sequence (11), it acts by multiplication by -1 also on $H_{\mathrm{dR}}^{1}(X / k)$.

Theorem 3.3. Let $p \geq 3$ and $a \in k^{\times}$, and let $q(z) \in k[z]$ be a monic polynomial of odd degree without repeated roots. Let $X$ denote the hyperelliptic curve given by the equation $y^{2}=f(x)$ where $f(x)=q\left(x^{p}-a^{p-1} x\right)$ and let $G$ denote the subgroup of $\operatorname{Aut}(X)$ generated by the automorphism $\tau$ given by $(x, y) \mapsto(x+a, y)$. Then the Hodge-de-Rham short exact sequence (11) does not split as a sequence of $k[G]$-modules.

\section{Remark 3.4.}

(a) Note that the polynomial $f(x)=q\left(x^{p}-a^{p-1} x\right) \in k[x]$ obviously has no repeated roots and thus $y^{2}=f(x)$ indeed defines a hyperelliptic curve.

(b) When applied to $q(z)=z$ and $a=1$, Theorem 3.3 becomes the main theorem of [Hor12].

(c) Theorem 3.3 implies that, for every algebraically closed field $k$ of characteristic $p \geq 3$, there exist infinitely many $g \geq 2$ and hyperelliptic curves over $k$ of genus $g$ for which the Hodge-de-Rham spectral sequence does not split equivariantly.

(d) Suppose $g \geq 2$ is given. If $p$ is a prime divisor of $2 g+1$ then, according to Theorem 3.3, every polynomial $q(z) \in k[z]$ of degree $(2 g+1) / p$ without repeated roots defines a hyperelliptic curve $X$ of genus $g$ for which the Hodge-de-Rham sequence does not split equivariantly.

Proof (of Theorem 3.3). We suppose that the sequence (11) does split and that

$$
s: H^{1}\left(X, \mathcal{O}_{X}\right) \rightarrow H_{\mathrm{dR}}^{1}(X / k)
$$

is a $k[G]$-linear splitting map. Then we have

$$
s\left(\tau^{*}(\alpha)\right)=\tau^{*}(s(\alpha)) \in H_{\mathrm{dR}}^{1}(X / k)
$$

and

$$
p(s(\alpha))=\alpha
$$

for all $\alpha \in H^{1}\left(X, \mathcal{O}_{X}\right)$. We will show that these equalities give rise to a contradiction when $\alpha$ is the residue class $\left[\frac{y}{x^{g}}\right]$ in $H^{1}\left(X, \mathcal{O}_{X}\right)$ (see Proposition 1.5). 
We first show that $\left[\frac{y}{x^{g}}\right] \in H^{1}\left(X, \mathcal{O}_{X}\right)$ is fixed by $\tau^{*}$. To this end, we recall that $\mathcal{U}=\left\{U_{0}, U_{\infty}\right\}, \mathcal{U}^{\prime}=\left\{U_{a}, U_{\infty}\right\}$ and $\mathcal{U}^{\prime \prime}=\left\{U_{0}, U_{a}, U_{\infty}\right\}$ and consider the following obviously commutative diagram of isomorphisms where $\rho$ and $\rho^{\prime}$ are defined as in (18):

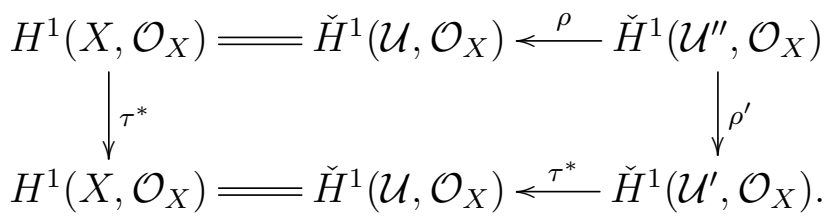

By Proposition 2.3 we have

$$
\begin{aligned}
\rho^{-1}\left(\left[\frac{y}{x^{g}}\right]\right) & =\left[\left(\frac{r_{g}(x) y}{x^{g}(x-a)^{g}}, \frac{y}{x^{g}}, \frac{t_{g}(x) y}{x^{g}(x-a)^{g}}\right)\right] \\
& =\left[\left(\frac{\left((x-a)^{g}-x^{g}\right) y}{x^{g}(x-a)^{g}}, \frac{y}{x^{g}}, \frac{y}{(x-a)^{g}}\right)\right] \text { in } \check{H}^{1}\left(\mathcal{U}^{\prime \prime}, \mathcal{O}_{X}\right) .
\end{aligned}
$$

We therefore obtain

$$
\begin{aligned}
\tau^{*}\left(\left[\frac{y}{x^{g}}\right]\right) & =\tau^{*}\left(\rho^{\prime}\left(\rho^{-1}\left(\left[\frac{y}{x^{g}}\right]\right)\right)\right) \\
& =\tau^{*}\left(\rho^{\prime}\left(\left[\left(\frac{\left.(x-a)^{g}-x^{g}\right) y}{x^{g}(x-a)^{g}}, \frac{y}{x^{g}}, \frac{y}{(x-a)^{g}}\right)\right]\right)\right) \\
& =\tau^{*}\left(\left[\frac{y}{(x-a)^{g}}\right]\right)=\left[\frac{y}{x^{g}}\right]
\end{aligned}
$$

as claimed.

By Theorem 2.2, the elements $\lambda_{i}, i=0, \ldots, g-1$, defined in (13) together with the elements $\gamma_{i}, i=1, \ldots, g$, defined in (12) form a basis of $H_{\mathrm{dR}}^{1}(X / k)$. Since the canonical projection $p: H_{\mathrm{dR}}^{1}(X / k) \rightarrow H^{1}\left(X, \mathcal{O}_{X}\right)$ is $k[G]$-linear and maps $\gamma_{g}$ to the residue class $\left[\frac{y}{x^{g}}\right]$, it follows that

$$
\tau^{*}\left(\gamma_{g}\right)=\gamma_{g}+\sum_{i=0}^{g-1} c_{i} \lambda_{i}
$$

for some $c_{0}, \ldots, c_{g-1} \in k$. On the other hand, we have

$$
s\left(\left[\frac{y}{x^{g}}\right]\right)=\gamma_{g}+\sum_{i=0}^{g-1} d_{i} \lambda_{i}
$$


for some $d_{0}, \ldots, d_{g-1} \in k$. Now the action of $\tau^{*}$ on $\lambda_{i}$ for $0 \leq i \leq g-1$ is easily seen to be given by

$$
\begin{aligned}
\tau^{*}\left(\lambda_{i}\right) & =\tau^{*}\left(\left[\left(\frac{x^{i}}{y} d x, \frac{x^{i}}{y} d x, 0\right)\right]\right) \\
& =\left[\left(\frac{(x+a)^{i}}{y} d x, \frac{(x+a)^{i}}{y} d x, 0\right)\right]=\sum_{k=0}^{i}\left(\begin{array}{l}
i \\
k
\end{array}\right) a^{i-k} \lambda_{k} .
\end{aligned}
$$

Plugging the equations obtained so far into equation 20 we obtain

$$
\begin{aligned}
\gamma_{g}+\sum_{i=0}^{g-1} d_{i} \lambda_{i} & =s\left(\left[\frac{y}{x^{g}}\right]\right)=s\left(\tau^{*}\left(\left[\frac{y}{x^{g}}\right]\right)\right) \\
& =\tau^{*}\left(s\left(\left[\frac{y}{x^{g}}\right]\right)\right)=\tau^{*}\left(\gamma_{g}+\sum_{i=0}^{g-1} d_{i} \lambda_{i}\right) \\
& =\left(\gamma_{g}+\sum_{i=0}^{g-1} c_{i} \lambda_{i}\right)+\sum_{i=0}^{g-1} d_{i}\left(\sum_{k=0}^{i}\left(\begin{array}{l}
i \\
k
\end{array}\right) a^{i-k} \lambda_{k}\right) .
\end{aligned}
$$

By comparing coefficients of the basis element $\lambda_{g-1}$, we see that $c_{g-1}=0$. On the other hand, we will below derive the equation $c_{g-1}=a / 4$ from the defining equation $(22)$. Since we assumed that $a \neq 0$, this gives us the desired contradiction.

The left-hand side of equation (22) is $\tau^{*}\left(\gamma_{g}\right)$. To compute $\tau^{*}\left(\gamma_{g}\right)$ we consider the following commutative diagram of isomorphisms where $\rho$ is the canonical projection (18) and $\rho^{\prime}$ is given by $\left(\omega_{0}, \omega_{a}, \omega_{\infty}, f_{0 a}, f_{0 \infty}, f_{a \infty}\right) \mapsto$ $\left(\omega_{a}, \omega_{\infty}, f_{a \infty}\right)$ :

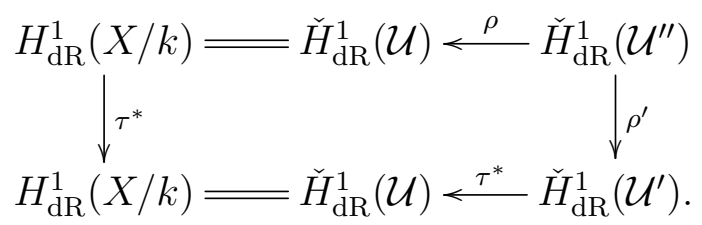

Then, by Proposition 2.3, we have:

$$
\begin{aligned}
\tau^{*}\left(\gamma_{g}\right) & =\tau^{*}\left(\rho^{\prime}\left(\rho^{-1}\left(\gamma_{g}\right)\right)\right) \\
& =\tau^{*}\left(\left[\omega_{a g}, \frac{-\phi_{g}(x)}{2 x^{g+1} y} d x, \frac{y}{(x-a)^{g}}\right)\right] \\
& =\left[\left(\tau^{*}\left(\omega_{a g}\right), \frac{-\phi_{g}(x+a)}{2(x+a)^{g+1} y} d x, \frac{y}{x^{g}}\right)\right] .
\end{aligned}
$$


On the other hand, the right hand side of equation 22 is equal to

$$
\left[\left(\frac{\psi_{g}(x)}{2 x^{g+1} y} d x, \frac{-\phi_{g}(x)}{2 x^{g+1} y} d x, \frac{y}{x^{g}}\right)\right]+\sum_{i=0}^{g-1} c_{i}\left[\left(\frac{x^{i}}{y} d x, \frac{x^{i}}{y} d x, 0\right)\right] .
$$

Note that the third entry in both (25) and (26) is $\frac{y}{x^{g}}$. Since any element of the form $\left(\omega_{0}, \omega_{\infty}, 0\right)$ in the subspace (8) of the space (7) in fact vanishes, the triples in (25) and (26) are equal already before taking residue classes. By comparing the second entries of (25) and (26) we therefore obtain the equation

$$
-\frac{\phi_{g}(x+a)}{2(x+a)^{g+1} y} d x=-\frac{\phi_{g}(x)}{2 x^{g+1} y} d x+\sum_{i=0}^{g-1} c_{i} \frac{x^{i}}{y} d x \quad \text { in } \quad \Omega_{K(X)} .
$$

Since $d x$ is a basis of $\Omega_{K(X)}$ considered as a $K(X)$-vector space, the equation above is equivalent to the equation

$$
\frac{\phi_{g}(x+a)}{2(x+a)^{g+1}}=\frac{\phi_{g}(x)}{2 x^{g+1}}-\sum_{i=0}^{g-1} c_{i} x^{i} \quad \text { in } \quad k(x),
$$

which in turn is equivalent to the equation

$$
\phi_{g}(x+a) x^{g+1}=\phi_{g}(x)(x+a)^{g+1}-2(x+a)^{g+1} x^{g+1} \sum_{i=0}^{g-1} c_{i} x^{i} \quad \text { in } \quad k[x] .
$$

Now, the assumption that the degree of $q(z)$ and hence also that of $f(x)$ is odd means that the degree of $f(x)$ is precisely $2 g+1$. By definition, the terms of highest degree in $\phi_{g}(x)$ are the same as the terms of highest degree in

$$
s_{g}(x)=x f^{\prime}(x)-2 g f(x)=x^{2 g+1}+0 \cdot x^{2 g}+\ldots .
$$

We therefore have

$$
\begin{aligned}
& \left((x+a)^{2 g+1}+0 \cdot(x+a)^{2 g}+\ldots\right) x^{g+1} \\
& \quad=\left(x^{2 g+1}+0 \cdot x^{2 g}+\ldots\right)(x+a)^{g+1}-2(x+a)^{g+1} x^{g+1}\left(c_{g-1} x^{g-1}+\ldots\right) .
\end{aligned}
$$

Hence, by comparing the coefficients of $x^{3 g+1}$, we obtain

$$
(2 g+1) a=(g+1) a-2 c_{g-1} .
$$


and hence

$$
c_{g-1}=\frac{((g+1)-(2 g+1)) a}{2}=-\frac{g}{2} a .
$$

Finally, we have $2 g+1=\operatorname{deg}(f(x))=\operatorname{deg}(q(z)) \cdot p \equiv 0 \bmod p$ and hence

$$
c_{g-1}=\frac{a}{4},
$$

as claimed above. This concludes the proof of Theorem 3.3 .

The first sentence of the following lemma provides a different way of stating the assumptions of Theorem 3.3. Although these alternative assumptions appear to be more general at first glance, the lemma states that they are actually essentially equivalent.

We return to our global assumptions that $X$ is an arbitrary hyperelliptic curve over $k$. Recall that the hyperelliptic involution $\sigma$ belongs to the centre of $\operatorname{Aut}(X)$ (see [Liu02, Corollary 7.4.31]). Then, given any $\tau \in \operatorname{Aut}(X)$, we have the induced map $\bar{\tau}: \mathbb{P}_{k}^{1} \rightarrow \mathbb{P}_{k}^{1}$, since $\mathbb{P}_{k}^{1}$ is the quotient of $X$ by the hyperelliptic involution. The following commutative diagram visualises this situation:

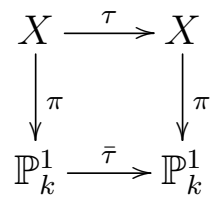

Lemma 3.5. Let $p \geq 3$ and suppose there exists an automorphism $\tau$ of $X$ such that $\bar{\tau}: \mathbb{P}_{k}^{1} \rightarrow \mathbb{P}_{k}^{1}$ is given by $x \mapsto x+a$ for some $0 \neq a \in k$. Then the action of $\tau^{*}$ on $y$ is given by $\tau^{*}(y)=y$ or $\tau^{*}(y)=-y$ and $f(x)$ is of the form $f(x)=q\left(x^{p}-a^{p-1} x\right)$ for some polynomial $q \in k[z]$.

Proof. We first show that $\tau^{*}(y)= \pm y$. There exist $g_{1}(x)$ and $g_{2}(x) \neq 0$ in $k(x)$ such that

$$
\tau^{*}(y)=g_{1}(x)+g_{2}(x) y \in k(x, y) .
$$

Hence

$$
f(x+a)=\tau^{*}\left(y^{2}\right)=\left(\tau^{*}(y)\right)^{2}=g_{1}(x)^{2}+2 g_{1}(x) g_{2}(x) y+g_{2}(x)^{2} f(x) .
$$

This implies that $g_{1}(x)=0$ because otherwise

$$
y=\frac{f(x+a)-g_{1}(x)^{2}-g_{2}(x)^{2} f(x)}{2 g_{2}(x) g_{1}(x)}
$$


would belong to $k(x)$. By comparing the degrees in (27) we see that $g_{2}(x)$ is a constant, and then by comparing coefficients in the same equation we see that $g_{2}(x)^{2}=1$. Hence $\tau^{*}(y)= \pm y$, as claimed.

We now show that $f(x)$ is of the form $q\left(x^{p}-a^{p-1} x\right)$. The extension $k(x)=k(z, x)$ of the rational function field $k(z)$ obtained by adjoining an element $x$ satisfying the equation $x^{p}-a^{p-1} x-z=0$ is a Galois extension with cyclic Galois group generated by the automorphism $x \mapsto x+a$. We derived above that $f(x)=f(x+a)$. Hence $f(x)$ belongs to $k(z)$. Furthermore, $x$ and hence $f(x)$ is integral over $k[z]$. Therefore, $f(x) \in k(z)$ belongs to $k[z]$, i.e., $f(x)=q\left(x^{p}-a^{p-1} x\right)$ for some $q \in k[z]$, as was to be shown.

We conclude with an example which demonstrates that the requirement in Theorem 3.3 for $q(z)$ to be of odd degree is a necessary condition.

Example 3.6. Let $p=3$ and $X$ be the hyperelliptic curve defined by the equation

$$
y^{2}=f(x)=x^{6}+x^{4}+x^{2}+2 .
$$

As in Theorem 3.3 let $\tau$ denote the automorphism of $X$ given by $(x, y) \mapsto$ $(x+1, y)$ and let $G:=\langle\tau\rangle$.

By Theorem 2.2, a basis of $\check{H}_{\mathrm{dR}}^{1}(\mathcal{U})$ is given by

$$
\begin{gathered}
\lambda_{0}=\left[\left(\frac{1}{y} d x, \frac{1}{y} d x, 0\right)\right], \quad \lambda_{1}=\left[\left(\frac{x}{y} d x, \frac{x}{y} d x, 0\right)\right], \\
\gamma_{1}=\left[\left(\frac{1}{x^{2} y} d x, \frac{x^{4}+2 x^{2}}{y} d x, \frac{y}{x}\right)\right], \quad \gamma_{2}=\left[\left(\frac{x^{2}+1}{2 x^{3} y} d x, \frac{2 x^{3}}{y} d x, \frac{y}{x^{2}}\right)\right] .
\end{gathered}
$$

By Proposition 1.5, the residue classes $\bar{\gamma}_{1}:=\left[\frac{y}{x}\right]$ and $\bar{\gamma}_{2}:=\left[\frac{y}{x^{2}}\right]$ form a basis of $H^{1}\left(X, \mathcal{O}_{X}\right)$. We define a map

$$
s: H^{1}\left(X, \mathcal{O}_{X}\right) \rightarrow H_{\mathrm{dR}}^{1}(X / k)
$$

of vector spaces over $k$ by

$$
\bar{\gamma}_{1} \mapsto \gamma_{1} \quad \text { and } \quad \bar{\gamma}_{2} \mapsto \gamma_{2}+\lambda_{1}
$$

Clearly $p \circ s$ is the identity map on $H^{1}\left(X, \mathcal{O}_{X}\right)$, and hence, if $s$ is $k[G]$-linear, the sequence in Proposition 2.1 does split as a sequence of $k[G]$-modules. 
We now show that $s$ is $k[G]$-linear. By Proposition 2.3, the pre-images of $\gamma_{1}$ and $\gamma_{2}$ under $\rho$ in $\check{H}_{\mathrm{dR}}^{1}\left(\mathcal{U}^{\prime \prime}\right)$ are the residue classes of

$$
\nu_{1}=\left(\frac{1}{x^{2} y} d x, \frac{x^{4}+2 x^{3}+2 x^{2}}{2(x-1)^{3} y} d x, \frac{x^{4}+2 x^{2}}{y} d x, \frac{y}{x(x-1)^{2}}, \frac{y}{x}, \frac{(x+1) y}{(x-1)^{2}}\right)
$$

and

$$
\nu_{2}=\left(\frac{x^{2}+1}{2 x^{3} y} d x, \frac{x^{3}+x^{2}+x+1}{2(x-1)^{3} y} d x, \frac{2 x^{3}}{y} d x, \frac{(x+1) y}{x^{2}(x-1)^{2}}, \frac{y}{x^{2}}, \frac{y}{(x-1)^{2}}\right),
$$

respectively. Using a computation similar to (25), it is easy to verify that

$$
\begin{aligned}
\tau^{*}\left(\gamma_{1}\right) & =\tau^{*}\left(\rho^{\prime}\left(\nu_{1}\right)\right) \\
& =\left[\left(\frac{x^{4}+2 x^{2}+2 x+2}{2 x^{3} y} d x, \frac{x^{4}+x^{3}+2 x^{2}+2 x}{y} d x, \frac{(x+2) y}{x^{2}}\right)\right] \\
& =\gamma_{1}+2 \gamma_{2}+2 \lambda_{1}
\end{aligned}
$$

and that

$$
\begin{aligned}
\tau^{*}\left(\gamma_{2}\right) & =\tau^{*}\left(\rho^{\prime}\left(\nu_{2}\right)\right) \\
& =\left[\left(\frac{x^{3}+x^{2}+1}{2 x^{3} y} d x, \frac{2 x^{3}+2}{y} d x, \frac{y}{x^{2}}\right)\right] \\
& =\gamma_{2}+2 \lambda_{0} .
\end{aligned}
$$

Furthermore, we have seen in 23 that

$$
\tau^{*}\left(\lambda_{0}\right)=\lambda_{0} \quad \text { and } \quad \tau^{*}\left(\lambda_{1}\right)=\lambda_{1}+\lambda_{0}
$$

We finally conclude that

$$
s\left(\tau^{*}\left(\bar{\gamma}_{1}\right)\right)=s\left(\bar{\gamma}_{1}+2 \bar{\gamma}_{2}\right)=\gamma_{1}+2 \gamma_{2}+2 \lambda_{1}=\tau^{*}\left(\gamma_{1}\right)=\tau^{*}\left(s\left(\bar{\gamma}_{1}\right)\right)
$$

and

$$
s\left(\tau^{*}\left(\bar{\gamma}_{2}\right)\right)=s\left(\bar{\gamma}_{2}\right)=\gamma_{2}+\lambda_{1}=\tau^{*}\left(\gamma_{2}+\lambda_{1}\right)=\tau^{*}\left(s\left(\bar{\gamma}_{2}\right)\right) .
$$

Hence $s$ is $k[G]$-linear, and the Hodge-de-Rham short exact sequence (11) splits. 
Remark 3.7. The curve $X$ defined in the previous example is isomorphic to the modular curve $X_{0}(22)$.

To see this, we first note that, by [KY10, Table 1], the modular curve $X_{0}(22)$ is the hyperelliptic curve of genus 2 defined by

$$
y^{2}=f(x)=x^{6}+2 x^{4}+x^{3}+2 x^{2}+1 .
$$

Now, $x \mapsto x-1, y \mapsto y$ defines an isomorphism between $X_{0}(22)$ and the curve defined by $y^{2}=x^{6}+2 x^{4}+2 x^{2}+2$. We finally apply the isomorphism described in the following general procedure.

If $g(x)=a_{s} x^{s}+\ldots+a_{0}$, and $a_{0} \neq 0 \neq a_{s}$, we define $g^{*}(x):=a_{0}^{-1} x^{s} g\left(\frac{1}{x}\right)$. It is stated after Lemma 2.6 in [KY10] that, if $y^{2}=g(x)$ defines a hyperelliptic curve and $s$ is even, then the curves defined by $y^{2}=g(x)$ and $y^{2}=g^{*}(x)$ are isomorphic.

\section{References}

[EP13] A. Elkin and R. Pries, Ekedahl-Oort strata of hyperelliptic curves in characteristic 2, Algebra Number Theory 7 (2013), no. 3, 507-532.

[Gro61] A. Grothendieck, Éléments de géométrie algébrique. III. Étude cohomologique des faisceaux cohérents. I, Inst. Hautes Études Sci. Publ. Math. (1961), no. 11.

[Hor12] Ruthi Hortsch, On the canonical representation of curves in positive characteristic, New York J. Math. 18 (2012), 911-924.

[KT15] B. Köck and J. Tait, Faithfulness of actions on Riemann-Roch spaces, Canad. J. Math. 67 (2015), no. 4, 848-869.

[KY10] A. Kontogeorgis and Y. Yang, Automorphisms of hyperelliptic modular curves $X_{0}(N)$ in positive characteristic, LMS J. Comput. Math. 13 (2010), 144-163.

[Liu02] Q. Liu, Algebraic geometry and arithmetic curves, Oxford Graduate Texts in Mathematics, vol. 6, Oxford University Press, Oxford, 2002, Translated from the French by Reinie Erné, Oxford Science Publications.

[Ma78] D. J. Madden, Arithmetic in generalized Artin-Schreier extensions of $k(x)$, J. Number Theory 10 (1978), no. 3, 303-323.

[Sti93] H. Stichtenoth, Algebraic function fields and codes, Universitext. SpringerVerlag, Berlin, 1993.

[Su75] F. J. Sullivan, p-torsion in the class group of curves with too many automorphisms, Arch. Math. (Basel) 26 (1975), 253-261. 
[Tai14] J. Tait, Group actions on differentials of curves and cohomology bases of hyperelliptic curves, $\mathrm{PhD}$ thesis, University of Southampton, 2014.

[Wed08] T. Wedhorn, De Rham cohomology of varieties over fields of positive characteristic, Higher-dimensional geometry over finite fields, 269-314, NATO Sci. Peace Secur. Ser. D Inf. Commun. Secur., 16, IOS, Amsterdam, 2008. 